\title{
Contemporary Results of Focal Therapy for Prostate Cancer Using Cryoablation
}

\author{
Vladimir Mouraviev, M.D., Ph.D.,' Truls Erik Bjerklund Johansen, M.D., Ph.D., \\ and Thomas J. Polascik, M.D.
}

\begin{abstract}
The concept of focal therapy is rapidly evolving and gaining popularity from both physician and patient perspectives. We review the rationale, candidate selection, and results of the first clinical studies of focal cryoablation for selected patients with low volume and low- to low-moderate-risk features of prostate cancer as an alternative to whole-gland treatment. In spite of improved understanding of the tumor biology of early stage disease, we currently have limited tools to select appropriate patients with low- to low-moderate risk unifocal or unilateral prostate cancer who may be amenable to focal therapy. From a technical point, a number of ablative treatment options for focal therapy are available, with cryoablation having the most clinical experience. Recently, several reports have been published from single and multi-institutional studies that discuss focal therapy as a reasonable balance between cancer control and quality-of-life outcomes. Retrospective pathologic data from large prostatectomy series, however, do not clearly reveal valid and reproducible criteria to select appropriate candidates for focal cryoablation because of the complexity of tumorigenesis in early stage disease. At this time, a more feasible option remains hemiablation of the prostate with reasonable certainty about the absence of clinically significant cancer lesion(s) on the contralateral side of the prostate based on three-dimensional transperineal prostate biopsy mapping studies. Minimally invasive, parenchyma-preserving cryoablation can be considered as a potential feasible option in the treatment armamentarium of early stage, localized prostate cancer in appropriately selected candidates. There is a need to further test this technique in randomized, multicenter clinical trials.
\end{abstract}

\section{Introduction}

$\mathbf{F}$ OR THE MOST PART, we still dogmatically consider prostate cancer ( $\mathrm{PCa}$ ) to be a heterogeneous, multifocal disease and thus treat patients with whole-gland therapy. The widespread screening programs and improved technique of prostate biopsy, however, led to the recent trend that approximately $90 \%$ of prostate cancers detected today are clinically localized. The uniqueness of these data are impressive compared with the clinically localized rate at the time of detection of other common cancers, such as breast $(63 \%)$, cervical $(55 \%)$, and colorectal $(39 \%)$. Furthermore, because a proportion of PCas now being diagnosed are unifocal, unilateral, or of lower malignant potential ("clinically insignificant"), this has raised questions as to whether all patients need radical treatment. ${ }^{1-6}$

According to CaPSURE data, the proportion of patients with favorable or low-risk tumor characteristics has risen from $29.8 \%$ in 1989 to 1992 to $45.3 \%$ in 1999 to 2001 . Moreover, this tendency is being accelerated today with the implementation of extended and multicore prostate biopsy. Despite the growing popularity of the concept of focal therapy, however, the absolute majority of low-risk patients according to the $\mathrm{D}^{\prime}$ Amico definition ${ }^{7}$ remain overtreated undergoing whole-gland therapy. Miller and associates 8 suggested that among the 24,405 men with lower-risk PCa, $55 \%$ received initial curative treatment; $10 \%$ were overtreated with radical prostatectomy and $45 \%$ with radiation therapy.

Ultimately, the treatment paradigm of managing earlystage, localized PCa is to distinguish patients who harbor lowrisk cancer for an active surveillance approach from those who might have a potentially dangerous index lesion that can be ablated and those who have life-threatening bilateral cancer necessitating a whole-gland radical treatment.

This review evaluates the rationale, candidate selection, and results of the first clinical studies of focal cryoablation for select patients to offer a "middle ground" treatment option between active surveillance and whole gland treatment.

\footnotetext{
${ }^{1}$ Division of Urologic Surgery and Duke Prostate Center, Department of Surgery, Duke University Medical Center, Durham, North Carolina.

${ }^{2}$ Department of Urology, Århus University Hospital, Århus, Denmark.
} 


\section{Evidence-Based Decisions}

A consensus panel of the 2nd Duke-Amsterdam International Symposium on Imaging and Focal Therapy recommended the following definition: "Focal therapy is a type of treatment that aims to eradicate known cancer within the prostate and at the same time preserve uninvolved prostatic tissue with the aim of preserving genito-urinary function." ${ }^{9}$

Accordingly, the goals of modern focal cryoablation include the following critical points:

1. Accurately eradicate known areas of cancer.

2. Induce local toxicity.

- Avoid collateral damage of surrounding anatomic structures.

- Avoid long- term complications.

3. Avoid systemic toxicity.

4. Fast and simple procedure.

The American Urological Association best practices policy statement summarized that subtotal prostate cryosurgery currently has level III evidence. While this concept is attractive from both a patient and physician perspective, clinical results are limited to short- or intermediate-term follow-up. Therefore, the panel's recommendation encouraged the collection of additional experience of subtotal prostate cryoablation for a more robust, evidence-based analysis.

Advantages of cryoablation compared with other minimally invasive technologies include its Food and Drug Administration approval for the management of localized PCa, technical development of third-generation cryotechnology that allows the performance of targeted freezing using ultrathin cryoprobes, and a minimal complication rate.

\section{Patient Selection}

Available selection criteria of ongoing clinical studies using focal cryoablation reported in the literature were mainly drawn from theory (Table 1). ${ }^{10-14}$ As a historical control, patient selection and prognostic indicators for radical prostatectomy (RP) have been aided by use of the Partin tables ${ }^{15}$ or Kattan nomogram ${ }^{16}$ that provides probabilities for adverse pathologic features, such as extraprostatic extension, seminal vesicle or lymph node involvement, as well as the likelihood for local or systemic recurrence. In contrast, there are no similar selection criteria for focal therapy because of the complexity of early-stage carcinogenesis.

Several investigators have used the $\mathrm{D}^{\prime}$ Amico definition ${ }^{7}$ to select appropriate candidates for focal therapy among lowrisk candidates. ${ }^{4,10-12}$ For example, in a clinical study of 538 patients with low- to low-moderate risk-features who were treated with RP, Polascik and colleagues ${ }^{17}$ found that only two pretreatment clinical variables were significantly predictive of pathologic unilateral PCa: Negative family history of PCa and clinical unilaterality based on prostate biopsy. The strongest predictor in multivariate analysis of pathologic unilaterality was biopsy unilaterality. These variables can be used to assess men with low- to low-moderate-risk PCa along with other established low-risk features before selection for subtotal ablation. Iczkowski and coworkers ${ }^{18}$ matched biopsy, RP, and clinical data from 393 patients at two institutions, concluding that only unilateral and unifocal cancer on prostate biopsy were strong predictors of unilateral and unifocal disease, respectively, for small-volume PCa $(\leq 0.5 \mathrm{cc})$. To date, it does not appear that any prostate-specific antigen (PSA) threshold, biopsy Gleason grade, or clinical stage cut point within the low-risk category can more reliably select men with unilateral or unifocal disease. ${ }^{17-19}$

At present, however, there is neither a consensus as to a strict definition of inclusion/exclusion criteria nor pretreatment planning guidance for focal therapy. For instance, Yoon and colleagues, ${ }^{20}$ analyzing the pathologic features of 100 RP specimens of low-risk patients with clinically unilateral disease by routine, office-based prostate biopsy, demonstrated that none of the seven patients with PSA levels higher than $10 \mathrm{ng} / \mathrm{mL}$ had clinically significant PCa in the contralateral lobe on RP pathology findings. Whether this finding implies that the baseline PSA level needs to be $<10 \mathrm{ng} / \mathrm{mL}$ to consider focal therapy remains unclear and needs further study.

Given the absence of an accurate imaging modality for PCa detection, the prostate biopsy remains the single crucial factor for treatment planning, especially when considering focal therapy. ${ }^{18,21-24}$ The spectrum of prostate biopsy cores taken ranges from 6 to $80+$, yet it is not known how many cores are

Table 1. Current Selection Criteria for Focal Cryoablation

\begin{tabular}{|c|c|c|c|c|}
\hline Study & $P S A n g / m L$ & GS & $c T$ & $b x$ criteria \\
\hline Bahn et $\mathrm{al}^{10 a}$ & All $($ mean $=4.9)$ & $5-7$ & $\mathrm{~N} / \mathrm{A}$ & $\begin{array}{l}\text { Initial } 6-8 \text { cores, then targeted bx } \\
\text { including NVB and SV if ECE } \\
\text { was suspected }\end{array}$ \\
\hline Ellis et $\mathrm{al}^{13}$ & All $($ mean $=7.2)$ & $3-10($ mean $=6)$ & $\mathrm{T}_{1 \mathrm{c}}-\mathrm{T}_{2 \mathrm{c}}$ & Unknown (retrospective study) \\
\hline Onik et $\mathrm{al}^{11}$ & $0.9-18$ & $3-8$ & $\mathrm{~T}_{1 \mathrm{c}}-\mathrm{T}^{2 \mathrm{~b}}$ & 2nd bx on negative side, TRUS \\
\hline Sartor et $\mathrm{al}^{14 \mathrm{~b}}$ & $\begin{array}{l}<10, \text { PSAD } \\
\quad<0.15 \mathrm{ng} / \mathrm{mL} / \mathrm{g}\end{array}$ & $\begin{array}{l}3+3 \text { or less } \\
\quad(\text { no grade } 4 \text { or } 5)\end{array}$ & $\begin{array}{l}\mathrm{T}_{1} \mathrm{~N}_{\mathrm{x}} \mathrm{M}_{\mathrm{x}} \\
\quad \text { or } \mathrm{T}_{2 \mathrm{a}} \mathrm{N}_{\mathrm{x}} \mathrm{M}_{\mathrm{x}}\end{array}$ & $\begin{array}{l}\text { Total length of PCa }<10 \mathrm{~mm} \text { total } \\
\text { and }<7 \mathrm{~mm} \text { in any } 1 \text { core; }<1 / 3 \\
\text { of cores positive for cancer. } 10 \\
\text { core minimum biopsy schema } \\
\text { plus two additional cores for } \\
\text { every } 10 \mathrm{~g} \text { of prostate }>40 \mathrm{~g} \\
\text { (maximum of } 18 \text { cores) }\end{array}$ \\
\hline
\end{tabular}

\footnotetext{
${ }^{a}$ No PSA, GS, cT selection criteria; required biopsy-proven, unilateral disease for entry.

${ }^{\mathrm{b}}$ Radiologic imaging (CT \pm MRSI): Largest dimension $<15 \mathrm{~mm}$ if prostate volume $>25 \mathrm{~g}$ or $<10 \mathrm{~mm}$ if volume $<25 \mathrm{~g}$. Capsular contact $<5 \mathrm{~mm}$ on axial imaging. No signs of ECE or SV invasion.

$\mathrm{CT}=$ computed tomography; $\mathrm{MRSI}=$ magnetic resonance spectroscopic imaging; $\mathrm{PSA}=$ prostate-specific antigen; $\mathrm{GS}=\mathrm{Gleason}$ score; $\mathrm{cT}=$ clinical stage; $\mathrm{bx}=$ biopsy; $\mathrm{N} / \mathrm{A}=$ not available; $\mathrm{NVB}=$ neurovascular bundle; $\mathrm{SV}=$ seminal vesicle; $\mathrm{ECE}=$ extracapsular extension; TRUS = transrectal ultrasonography; PSAD prostate-specific antigen density; $\mathrm{PCa}=$ prostate cancer.
} 
sufficient to assure that a clinically significant cancer will not be missed on the untreated side, ${ }^{21,22,24-27}$ Even a single positive core that is identified in low-risk PCa when obtained with routine transrectal biopsy techniques may not exclude bilateral disease in all instances. Barber and associates ${ }^{28}$ investigated the data of 129 men with a single positive PCa core (in a 6-12 core biopsy), with 46 (36\%) patients subsequently treated with RP. Final pathology assessment showed that almost $90 \%$ with Gleason score $\leq 6$ have disease on the side contralateral to the positive biopsy core and approximately one fourth $(22 \%)$ have a component of high-grade disease in the contralateral lobe. The single positive core correctly predicted the laterality of the largest tumor focus in $71 \%$ of cases. Emerging data suggest that the traditional sextant or extended 12-core biopsy is likely not sufficient to exclude contralateral disease, despite a single or unilateral focus that is identified by routine biopsy. $20,25,27$

Most of the literature that analyzes various biopsy schemes is aimed at the detection of PCa without regard to laterality or focality because this detailed information is not necessary for whole-gland therapy. ${ }^{23,29-31}$ Similarly, retrospective analysis of various biopsy techniques that are performed simply to diagnose and not map PCa foci will have limited applicability for focal therapy planning.

It appears that different biopsy techniques that positionally map PCa foci in three dimensions will be necessary for focal therapy. Crawford and Barqawi ${ }^{26}$ introduced templateguided transperineal, three-dimensional pathologic mapping biopsy (3DMB) to detect clinically significant cancers before focal therapy. In a computer-simulated study of RP and cadaveric specimens, they found that using 5-mm grid spacing to obtain a mapping biopsy appears to be more accurate to detect clinically significant cancers (85\% vs 37\%, respectively). From their recent update of 146 patients with low-risk features by the $\mathrm{D}^{\prime}$ Amico definition, 3DMB (mean number of cores 61.5) in comparison with TRUS- guided biopsy (mean number of cores 11.5) revealed that a significant portion of men harbor a clinically significant PCa that can facilitate optimal treatment planning for focal ablation. ${ }^{32}$ These results are encouraging for providing superior localization compared with routine TRUS biopsy. If confirmed with more data, this technique may become an essential component to select patients for focal therapy until an appropriate imaging modality is developed.

Several limitations in this field need to be outlined. One weak point in published series is a lack of standardization of pretreatment biopsies. Clinical protocols should be standardized for both the treated and untreated sides of the prostate with more details; eg, the localization of positive biopsies and their percent of tumor involvement. In addition, a certain number of biopsies have to be defined per $\mathrm{cm}^{3}$ of prostate tissue to ensure that clinically significant cancer is not inadvertently missed because of sampling limitations.

Ultimately, when selecting patients, we need to consider several critical questions about the possible goal(s) of focal therapy:

(1) Is the expectation cancer cure or cancer control?

(2) Is clinically significant PCa being inadvertently missed in the untreated part of the gland?

(3) How can careful surveillance of the untreated parenchyma be optimized?

\section{Current Protocols of Focal Cryoablation}

A nerve-sparing cryoablation technique to manage $\mathrm{PCa}$ was first described in 2002 by Onik and coworkers ${ }^{33}$ in a pilot study of nine patients by performing unilateral ablation of the lobe where the positive biopsy was identified and leaving the contralateral lobe and neurovascular bundle untreated. After a mean follow-up of 3 years, seven patients were potent. All patients had a stable PSA level and negative follow-up biopsies. Subsequent experimental data in a canine model, however, raised the question of incomplete ablation in the peripheral prostate tissue; ie, the most frequent location of PCa that can jeopardize cancer control. ${ }^{34}$ Therefore, subtotal glandular ablation did not gain popularity until recently when documented acceptable short- and intermediate-term cancer control and functional outcomes became available. ${ }^{10-12}$ The recent technical innovations using third-generation argon-helium gas systems are now capable of performing efficient and targeted cryoablation. ${ }^{35-39}$

As mentioned, focal therapy includes any type of subtotal glandular ablation that attempts to eliminate the cancer yet preserve uninvolved tissue while maintaining a patient's quality of life (QoL), such as erectile, urinary, and bowel function (Fig. 1). Focal therapy is intended to be a tailored treatment, thereby avoiding overtreatment while cautiously providing awareness of potential undertreatment. ${ }^{35,39-43}$

In the absence of image-guided therapy, at present, a practical approach is hemiablation of the prostate leaving untreated an intact lobe that will be able to facilitate an acceptable level of QoL, including potency and urinary continence. Retrospective analysis of a few large prostatectomy databases demonstrated that some proportion of the patients (up to 20\%) with low-risk PCa could be selected for hemiablation. While Tareen and associates ${ }^{44}$ stated that unilaterality alone should not drive selection of candidates for hemiablative focal therapy in their large comparative study of $1458 \mathrm{RP}$ series (311 with unilateral vs 1147 with bilateral tumors), these authors concluded at the same time that men with unilateral PCa have more favorable oncological outcomes than those with bilateral PCa. By collecting more data on the tumor biology of unilateral disease, the critical aspects of this entity may be gradually unveiled. For instance, Weckermann and coworkers ${ }^{45}$ investigated the data of 564 men with $\mathrm{cT}_{1 \mathrm{c}}$ or unilateral palpable disease with positive biopsy cores only in one lobe who underwent RP with some cases of pelvic node dissection. They found that unilateral PCa preferentially metastasized to the ipsilateral pelvic lymph nodes.

Therefore, at this stage of our knowledge, hemiablation can be considered as an optimal model for focal therapy as a next historic control (after active surveillance with organ preservation and RP) toward true focal ablation of unifocal lesions in the future. The urethra serves as the natural anatomic structure in the midline that allows sculpting of the ice ball within one lobe. A review of pathology data, however, both retrospectively and prospectively, can serve to suggest other alternative options for the development of the focal therapy concept. For instance, Ward and colleagues ${ }^{46}$ did a comparison between hemiablation and 3/4 ablation templates on the data of 180 men with unilateral disease who proceeded with RP. These two modalities would have successfully treated all clinically significant prostate tumors in $64 \%$ and $81 \%$ of patients, respectively. Thus, the $3 / 4$ template encompassed 


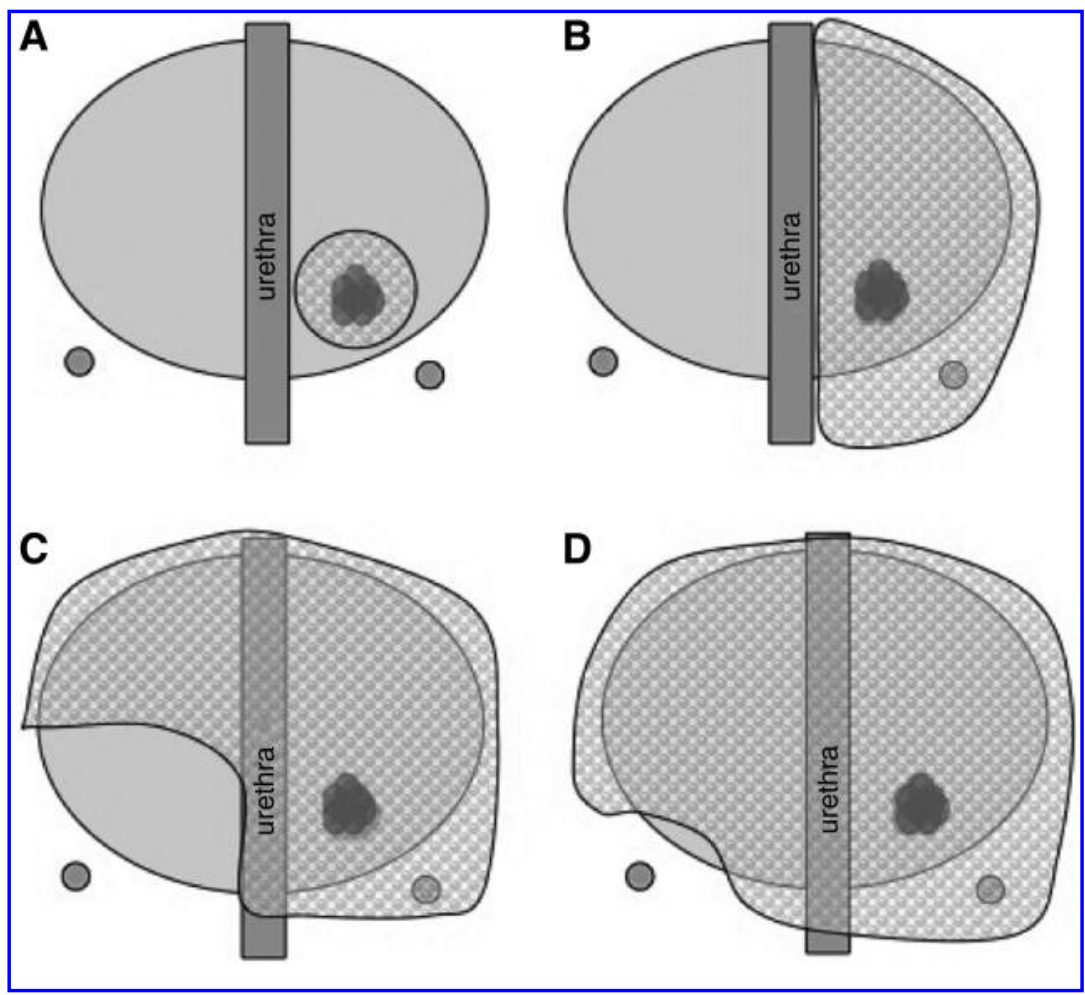

FIG. 1. Possible focal therapy options. (A) True focal ablation; (B) hemiablation; (C) 75\% ablation; (D) near total ablation.

almost all index lesions. These results, however, need to be reproduced by clinical data in men who are treated in such fashion.

\section{Clinical Application of Focal Targeted Cryoablation}

To date, several small studies have reported clinical results of focal cryoablation, including short- and intermediate-term data. ${ }^{10-13,47}$ Only one study explored a technique of focal targeted ablation of a unifocal lesion. Onik and associates ${ }^{47}$ recently updated their series of 48 patients with at least 2-year (mean 4.5 years) follow-up. In total, 45 of 48 (94\%) patients have stable PSA levels with no evidence for cancer, despite 25 patients being medium to high risk for recurrence. No local recurrences were noted in the areas that were treated based on biopsy findings. In 4 of 48 patients with an unstable PSA level within the first year of follow-up, biopsy findings revealed persistent tumor in the untreated area of the gland. Potency was maintained to the satisfaction of the patient in 36 of 40 men who were potent pretreatment. Of the 48 men in the series, all were continent after focal ablation.

Lambert and colleagues ${ }^{12}$ presented data on 25 patients who underwent unilateral cryoablation based on a minimum 12-core biopsy that suggested the presence of PCa in one lobe. The criteria for suspicion of biochemical failure after treatment was a PSA nadir less than $50 \%$. Of the 25 patients, $21(84 \%)$ demonstrated PSA disease-free survival defined as a greater than $50 \%$ PSA nadir reduction. Seven patients underwent repeated prostate biopsy with cancer detected in the contralateral untreated lobe in two $(8 \%)$ patients and in the treated lobe in one $(4 \%)$ patient. Of 24 patients who were potent preoperatively, 17 (71\%) were potent after treatment. All patients preserved their pretreatment urinary function, maintaining complete continence.

Bahn and coworkers ${ }^{10}$ reported the results of a twoinstitution trial of 31 patients with clinically organ-confined, unilateral cancer that was identified by color Doppler ultrasonography and confirmed by targeted and systematic biopsy. All patients, who had a strong desire for preservation of sexual function and continence, were treated with unilateral cryoablation. Cancer control data at a mean follow-up of 70 months were acceptable; eg, biochemical disease-free survival using the American Society for Therapeutic Radiology and Oncology (ASTRO) definition of three consecutive PSA increases ${ }^{48}$ was maintained in $26(92.8 \%)$ of 28 patients with negative biopsies in $24(96 \%)$ of 25 patients. The one patient with a positive biopsy in the apex of the contralateral untreated lobe was subsequently re-treated with full-gland therapy and remains disease free. The overall potencypreservation rate was $88.9 \%$. There were no cases of incontinence or other complications.

Ellis and associates ${ }^{13}$ presented a series of 60 consecutive patients with a median age of 69 years, median PSA level of $7.2 \mathrm{ng} / \mathrm{mL}$, median Gleason score of 6 , and median clinical stage of $\mathrm{T}_{1 \mathrm{c}}$ disease with a mean follow-up of $15.2 \pm 7.4$ months after focal unilateral cryoablation. Biochemical disease-free survival according to the ASTRO definition ${ }^{48}$ was $80.4 \%$ with a positive biopsy rate of $40 \%$ (with 13 of 14 cases positive on the untreated side). The authors stated that using a penile rehabilitation program, a potency rate of $70.6 \%$ was achieved within 12 months. A total of $3.6 \%$ of patients were incontinent at $\geq 6$ months. Interestingly, 11 patients under- 
went a second focal cryoablation because of persistent cancer. Of five patients with preserved erectile function after the first cryoablation treatment, all (100\%) regained potency after the second procedure.

At the 2009 American Urological Association meeting, Dhar and colleagues ${ }^{49}$ reported the results of 795 patients who were treated with subtotal prostate cryoablation from the Cryo On-Line Data registry. Patient characteristics were: Median age of 68 (37-85) years, median Gleason score of 6 (6-9), and median follow-up of 1 year. The positive biopsy rate after focal cryotherapy was $4.5 \%$ of the total population. The cancer control data at 5 year follow-up were superior in low- and intermediate-risk categories than in high-risk, especially per the Phoenix-definition ${ }^{50}$; eg, $64 \%$, 69\% vs $41 \%$, respectively (Table 2 ). Regarding complications, incontinence was identified in $2.8 \%$. Of the 134 patients who were potent before cryoablation, 87 (65\%) were sexually active 12 months postoperatively. Several limitations raise concerns about the design and quality of this large multicenter study; eg, why patients with high-risk features were included, how many biopsy cores were necessary for entry, the selection criteria used, and types of focal ablation. It is also not clear if the follow-up evaluation was standardized, including a specific post-treatment biopsy protocol.

\section{Possible Pitfalls and Concerns}

The essential argument for opponents of focal therapy is missing clinically significant PCa in the untreated part of the gland. In other words, to what extent will tumor control be jeopardized in favor of preserving sexuality? Rukstalis and coworkers $^{51}$ analyzed 112 RP specimens and concluded that if the index lesion (largest tumor) detected with biopsy could be extensively ablated in 9 of 12 prostate zones, cancer control would be accomplished with a $21 \%$ risk of significant (ie, $>0.5 \mathrm{cc}$ ) residual disease.

Yoon and colleagues ${ }^{20}$ assessed 100 RP specimens from patients with low-risk unilateral disease (based on routine

Table 2. Cancer Control and Complication Rates After Focal and Unilateral Cryoablation

\begin{tabular}{|c|c|c|c|c|c|c|}
\hline Reference & $\begin{array}{l}\text { No. } \\
\text { pts }\end{array}$ & Mean F/U & bDFS - No. (\%) & $\begin{array}{l}\text { Biopsy-proven } \\
\text { recurrence }\end{array}$ & $\begin{array}{c}\text { Potency } \\
\text { preserved }(\%)\end{array}$ & $\begin{array}{c}\text { Continence } \\
\text { preserved }(\%)\end{array}$ \\
\hline \multicolumn{7}{|c|}{ Focal targeted cryoablation of unifocal lesion } \\
\hline Onik et $\mathrm{al}^{11}$ & 55 & 3.6 yrs & $52(95 \%)$-ASTRO & $\begin{array}{l}4(7 \%) \text {-untreated } \\
\text { area }\end{array}$ & $44(85 \%)$ & $54(95 \%)$ \\
\hline \multicolumn{7}{|c|}{ Unilateral cryoablation } \\
\hline Bahn et $\mathrm{al}^{10}$ & 31 & $70 \mathrm{mos}$ & $26 / 28(93 \%)-A S T R O$ & $\begin{array}{l}1 / 25(4 \%) \text { - untreated } \\
\text { lobe }\end{array}$ & $\begin{array}{l}24 / 27(88.9 \%) \\
\text { totals: } \\
13 / 27(48.1 \%) \\
\text { fully recovered } \\
11 / 27(40.8 \%) \\
\text { medication } \\
\text { assisted }\end{array}$ & $31(100 \%)$ \\
\hline${\text { Lambert et } a 1^{12}}^{12}$ & 25 & $28 \mathrm{mos}$ & $\begin{array}{l}21(88 \%)-<50 \% \\
\text { PSA nadir }\end{array}$ & $\begin{array}{l}3(12 \%) \text { total: } \\
2(8 \%) \text { - untreated } \\
\text { lobe } \\
1(4 \%) \text { - treated lobe }\end{array}$ & $17(71 \%)$ & $25(100 \%)$ \\
\hline Ellis et al ${ }^{13}$ & 60 & $16.7 \mathrm{mos}$ & $42(80 \%)-A S T R O$ & $14(23 \%)$ total: & $\begin{array}{l}24 / 34(70.6 \%) \\
\text { with sexual } \\
\text { rehabilitation }\end{array}$ & $53 / 55(96.4 \%)$ \\
\hline & & & & $\begin{array}{l}13(22 \%) \text { - untreated } \\
\text { lobe } \\
1(1 \%) \text { - treated lobe }\end{array}$ & & \\
\hline \multicolumn{7}{|c|}{ All kinds of focal/partial gland cryoablation } \\
\hline Dhar et $\mathrm{al}^{49 \mathrm{a}}$ & 795 & 12 mos & $\begin{array}{l}\text { 5-year bDFS } \\
\text { (ASTRO): } \\
81.4 \% \text { - low-risk } \\
81.2 \% \text {-intermediate } \\
\text { risk } \\
\text { 83.4\% - high-risk } \\
\text { 5-year - bDFS } \\
\text { (Phoenix): } \\
\text { 63.9\% - low-risk } \\
\text { 68.7\% -intermediate-risk } \\
\text { 35\% - high-risk }\end{array}$ & 26 /199 (4.5\%) & $87 / 134(65 \%)$ & $11 / 393(97.2 \%)$ \\
\hline
\end{tabular}

\footnotetext{
${ }^{a}$ In three cases $(0.4 \%)$, rectal fistula was diagnosed between 6 weeks and 12 months postoperatively.

$\mathrm{Pts}=$ patients; $\mathrm{F} / \mathrm{U}=$ follow-up; ASTRO = American Society for Therapeutic Radiology and Oncology; bDFS=biochemical disease-free survival.
} 
transrectal biopsy findings) and found contralateral disease in 65 cases with 13 cases having tumor volumes more than $0.5 \mathrm{cc}$. They identified that the more frequent location of "hidden lesions" on the side contralateral to the positive biopsy were anteriorly in seven cases, with six specimens having PCa in the transition zone and one in the anterior horn of the peripheral zone and the transition zone. Ultimately, $20 \%$ of all cases had adverse pathology findings according to tumor size, extracapsular extension (ECE), grade, or positive surgical margins on the contralateral side.

Of 275 patients with clinically low-moderate risk features, Polascik and coworkers ${ }^{17}$ found that clinically significant PCa was identified contralateral to a unilaterally positive prostate biopsy (6-16 cores) in RP specimens that had such adverse pathologic features in $24 \%$ of all cases including ECE (14.9\%), percent of tumor involvement $>15 \%(8.4 \%)$, Gleason score $>7$ $(4.7 \%)$, seminal vesicle involvement $(2.5 \%)$, or its combination. Johnstone and colleagues ${ }^{52}$ found that the diagnostic pattern for the conventional prostate biopsy (6-12 cores) was unreliable compared with RP specimens in nine reported series comprising almost 800 cases with minimal unilateral disease by prostate biopsy. Final pathologic assessment of prostatectomy specimens revealed a maximal tumor volume $>10 \mathrm{cc}$ in three series, ECE in $10.5 \%$ of cases, a positive surgical margin rate of $10.5 \%$, Gleason 4 pattern in $14 \%$, and bilaterality in about $80 \%$, respectively.

When considering targeted focal ablation, the main focus at diagnosis is to determine: (1) the three-dimensional cancer location within the gland, (2) cancer volume, and (3) biologic potential (eg, which aggressive foci need immediate therapy, while other indolent foci may be able to be followed or treated with a chemoprevention strategy). The challenge is how to define the clinically significant PCa focus based on its natural biologic history tempered with patient life expectancy. Further research will be needed to understand the biologic potential of each of the individual PCa foci within a cancerous gland. In addition, chemoprevention combined with focal treatment is a future viable concept that deserves further exploration. $^{26}$

In the absence of randomized clinical trials, the short-term results of phase II studies (Table 2) demonstrated a promising balance between cancer control and QoL issues in carefully selected candidates with early-stage prostate cancer. While realizing not complete eradication of cancer in prostate after focal therapy with possible development of other tumor focus(i) in an untreated area, it remains of paramount importance to perform a thorough and regular active follow-up of this patients. Other serious limitations to evaluating the effectiveness of focal cryoablation on QoL issues is an absence of assessment tools with established and validated criteria or instruments to define sexual well-being and continence function. Historically, it has not been perfectly done for different kinds of whole-gland therapy, such as radical prostatectomy, radiation therapy, or cryoablation. Therefore, the development of these criteria reflecting treatment outcomes needs to be initiated as a first priority in the focal therapy setting.

\section{Conclusion}

One main achievement in the developing concept of focal therapy is the willingness of an interdisciplinary scientific community to steadily implement advances in imaging and ablative technologies into clinical practice. Although impediments to the widespread adoption of focal therapy exist, primarily because of the multifocal nature of $\mathrm{PCa}$ and the current lack of precise imaging, research into clinical predictors of PCa unifocality or unilaterality is necessary to development of the focal therapy concept.

A major research goal is the identification and development of reliable pretreatment prognostic indicators that can accurately predict the natural history of a patient's tumor. Significant advances in tumor biology and the introduction of novel minimally invasive therapies may further facilitate the inclusion of minimally invasive treatment options for selected candidates in contrast to radical whole-gland treatment as we move toward a more individually tailored therapy program. For those patients with unifocal or unilateral PCa lesions who seek a more targeted treatment with QoL preservation, options such as focal targeted cryoablation are currently available. Only long-term results of prospective multi-institutional clinical trials, however, will determine outcomes among patients with small-volume PCa and increase understanding of tumor biology.

\section{Disclosure Statement}

Dr. Polascik is a research consultant for Galil Medical. The other authors have no competing financial interests.

\section{References}

1. Bostwick DG, Waters DJ, Farley ER, et al. Group consensus reports from the Consensus Conference on Focal Treatment of Prostatic Carcinoma, Celebration, Florida, February 24, 2006. Urology 2007;70(suppl 6):42-44.

2. Meiers I, Waters DJ, Bostwick DG. Preoperative prediction of multifocal prostate cancer and application of focal therapy: Review 2007. Urology 2007;70(suppl 6):3-8.

3. Polascik TJ, Mouraviev V. Focal therapy for prostate cancer. Curr Opin Urol 2008;18:269-274.

4. Barqawi AB, Crawford ED. The current use and future trends of focal surgical therapy in the management of localized prostate cancer. Cancer J 2007;13:313-317.

5. Mouraviev V, Mayes JM, Sun L, et al. Prostate cancer laterality as a rationale of focal ablative therapy for the treatment of clinically localized prostate cancer. Cancer 2007; 110:906-910.

6. Polascik TJ, Mayes JM, Sun L, et al. Pathologic stage T2a and $\mathrm{T} 2 \mathrm{~b}$ prostate cancer in the recent prostate-specific antigen era: Implications for unilateral ablative therapy. Prostate 2008;68:1380-1386.

7. D'Amico AV, Moul J, Carroll PR, et al. Cancer-specific mortality after surgery or radiation for patients with clinically localized prostate cancer managed during the prostatespecific antigen era. J Clin Oncol 2003;21:2163-2172.

8. Miller DC, Gruber SB, Hollenbeck BK, et al. Incidence of initial local therapy among men with lower-risk prostate cancer in the United States. J Natl Cancer Inst 2006;98:1134-1141.

9. de la Rosette J, Ahmed H, Barentsz J, et al. Focal therapy in prostate cancer-report from a consensus panel. I Endourol 2010;24:775-780.

10. Bahn DK, Silverman P, Lee F Sr, et al. Focal prostate cryoablation: Initial results show cancer control and potency preservation. J Endourol 2006;20:688-692. 
11. Onik G, Vaughan D, Lotenfoe R, et al. "Male lumpectomy": Focal therapy for prostate cancer using cryoablation. Urology 2007;70(suppl 6):16-21.

12. Lambert EH, Bolte K, Masson P, Katz AE. Focal cryosurgery: Encouraging health outcomes for unifocal prostate cancer. Urology 2007;69:1117-1120.

13. Ellis DS, Manny TB Jr, Rewcastle JC. Focal cryosurgery followed by penile rehabilitation as primary treatment for localized prostate cancer: Initial results. Urology 2007;70 (suppl 6):9-15.

14. Sartor AO, Hricak H, Wheeler TM, et al. Evaluating localized prostate cancer and identifying candidates for focal therapy. Urology 2008;72(suppl 6):S12-S24.

15. Partin AW, Mangold LA, Lamm DM, et al. Contemporary update of prostate cancer staging nomograms (Partin Tables) for the new millennium. Urology 2001;58:843-848.

16. Kattan MW, Eastham JA, Stapleton AM, et al. A preoperative nomogram for disease recurrence following radical prostatectomy for prostate cancer. J Natl Cancer Inst 1998;90: 766-771.

17. Polascik TJ, Mayes JM, Schroeck FR, et al. Patient selection for hemiablative focal therapy of prostate cancer: Variables predictive of tumor unilaterality based upon radical prostatectomy. Cancer 2009;115:2104-2110.

18. Iczkowski KA, Hossain D, Torkko KC, et al. Preoperative prediction of unifocal, unilateral, margin-negative, and small volume prostate cancer. Urology 2008;71:1166-1171.

19. Tareen B, Sankin A, Godoy G, et al. Appropriate candidates for hemiablative focal therapy are infrequently encountered among men selected for radical prostatectomy in contemporary cohort. Urology 2009;73:351-355.

20. Yoon GS, Wang W, Osunkoya AO, et al. Residual tumor potentially left behind after local ablation therapy in prostate adenocarcinoma. J Urol 2008;179:2203-2206.

21. Andriole GL, Bullock TL, Belani JS, et al. Is there a better way to biopsy the prostate? Prospects for a novel transrectal systematic biopsy approach. Urology 2007;70(suppl 6):22-26.

22. Boccon-Gibod LM, de Longchamps NB, Toublanc M, et al. Prostate saturation biopsy in the reevaluation of microfocal prostate cancer. J Urol 2006;176:961-964.

23. Graser A, Heuck A, Sommer B, et al. Per-sextant localization and staging of prostate cancer: Correlation of imaging findings with whole-mount step section histopathology. AJR Am J Roentgenol 2007;188:84-90.

24. Onik G, Barzell W. Transperineal 3D mapping biopsy of the prostate: An essential tool in selecting patients for focal prostate cancer therapy. Urol Oncol 2008;26:506-510.

25. Barzell WE, Melamed MR. Appropriate patient selection in the focal treatment of prostate cancer: The role of transperineal 3-dimensional pathologic mapping of the prostate-a 4-year experience. Urology 2007;70(suppl 6):27-35.

26. Crawford ED, Barqawi A. Targeted focal therapy: A minimally invasive ablation technique for early prostate cancer. Oncology (Williston Park) 2007;21:27-39.

27. Mayes J, Mouraviev V, Sun L, et al. Can the conventional sextant prostate biopsy reliably diagnose unilateral prostate cancer in low-risk, localized, prostate cancer? Urol Oncol 2009; May 16. E-pub ahead of print.

28. Barber T, Pansare V, Nikolavsky D, et al. Pathologic characteristics of contralateral prostate cancer among patients with a single positive core. J Urol 2006;175(suppl);507. Abstract 1573.

29. Presti JC. Prostate biopsy: Current status and limitations. Rev Urol 2007;9:93-98.
30. Ravery V, Dominique S, Panhard X, et al. The 20-core prostate biopsy protocol—a new gold standard? J Urol 2008;179:504507.

31. Jones JS. Saturation biopsy for detecting and characterizing prostate cancer. BJU Int 2007;99:1340-1344.

32. Barqawi A, Arangua PR, ED C. The role of $3 \mathrm{D}$ mapping biopsy of the prostate in decision making for treatment of early stage prostate cancer. Abstracts of the American Urological Association annual meeting. April 25-30, 2009; Chicago, IL. J Urol 2009;181(suppl 4):717. Abstract 1979.

33. Onik G, Narayan P, Vaughan D, et al. Focal "nerve-sparing" cryosurgery for treatment of primary prostate cancer: A new approach to preserving potency. Urology 2002;60:109-114.

34. Janzen NK, Han KR, Perry KT, et al. Feasibility of nervesparing prostate cryosurgery: Applications and limitations in a canine model. J Endourol 2005;19:520-525.

35. Mouraviev V, Polascik TJ. Avoiding surgery in prostate cancer patients with low-risk disease. Future Medicine. Therapy 2008;5:25-36.

36. Cresswell J, Asterling S, Chaudhary M, et al. Thirdgeneration cryotherapy for prostate cancer in the UK: A prospective study of the early outcomes in primary and recurrent disease. BJU Int 2006;97:969-974.

37. Miller R, Cohen JJ. Prostate cryosurgery. In: Rukstalis DB, Katz AE, eds. Handbook of Urologic Cryoablation. London: Informa. UK Ltd. 2007:39-46.

38. Polascik TJ, Nosnik I, Mayes JM, Mouraviev V. Short-term cancer control after primary cryosurgical ablation for clinically localized prostate cancer using third-generation cryotechnology. Urology 2007;70:117-121.

39. Polascik TJ, Mayes JM, Mouraviev V. From whole-gland to targeted cryoablation for the treatment of unilateral or focal prostate cancer. Oncology (Williston Park) 2008;22:900-914.

40. Ahmed HU, Emberton M. Active surveillance and radical therapy in prostate cancer: Can focal therapy offer the middle way? World J Urol 2008;26:457-467.

41. Eggener SE, Scardino PT, Carroll PR, et al; International ask Force on Prostate Cancedr and the Focal Lesion Paradigm. Focal therapy for localized prostate cancer: A critical appraisal of rationale and modalities. J Urol 2007;178:22602267.

42. Jones JS. Focal or subtotal therapy for early stage prostate cancer. Curr Treat Options Oncol 2007;8:165-172.

43. Ahmed HU, Pendse D, Illing R, et al. Will focal therapy become a standard of care for men with localized prostate cancer? Nat Clin Pract Oncol 2007;4:632-642.

44. Tareen B, Godoy G, Sankin A, et al. Laterality alone should not drive selection of candidates for hemi-ablative focal therapy. I Urol 2009;181:1082-1090.

45. Weckermann D, Holl G, Dorn R, et al. Reliability of preoperative diagnostics and location of lymph node metastases in presumed unilateral prostate cancer. BJU Int 2007;99:10361040.

46. Ward JF, Nakanishi H, Pisters L, et al. Cancer ablation with regional templates applied to prostatectomy specimens from men who were eligible for focal therapy. BJU Int 2009;104: 490-497.

47. Onik G, Vaughan D, Lotenfoe R, et al. The "male lumpectomy": Focal therapy for prostate cancer using cryoablation results in 48 patients with at least 2-year follow-up. Urol Oncol 2008;26:500-505.

48. ASTRO. Consensus statement: Guidelines for PSA following radiation therapy. Int J Radiat Oncol Biol Phys 1997;37:10351041. 
49. Dhar N, Cher ML, Scionti SM, et al. Focal/partial gland prostate cryoablation: Results of 795 patients from multiple centers tracked with the COLD registry. Abstracts of the American Urological Association annual meeting. April 2530, 2009; Chicago, IL. J Urol 2009;181(suppl 4):715. Abstract 1975.

50. Roach M III, Hanks G, Thames H Jr, et al. Defining biochemical failure following radiotherapy with or without hormonal therapy in men with clinically localized prostate cancer: Recommendations of the RTOG-ASTRO Phoenix Consensus Conference. Int J Radiat Oncol Biol Phys 2006;65: 965-974.

51. Rukstalis DB, Goldknopf JL, Crowley EM, Garcia FU. Prostate cryoablation: A scientific rationale for future modifications. Urology 2002;60(suppl 1):19-25.

52. Johnstone PA, Rossi PJ, Jani AB, Master V. 'Insignificant' prostate cancer on biopsy: Pathologic results from subsequent radical prostatectomy. Prostate Cancer Prostatic Dis 2007;10:237-241.
Address correspondence to:

Thomas J. Polascik, M.D.

Division of Urologic Surgery

Duke University Medical Center

Box 2804 Yellow Zone

Durham, NC 27710

E-mail: polas001@mc.duke.edu

\begin{aligned} & \multicolumn{1}{c|}{ Abbreviations Used } \\ & ASTRO $=$ American Society for Therapeutic Radiology and \\ & Oncology \\ & $\mathrm{ECE}=$ extracapsular extension \\ & $\mathrm{PCa}=$ prostate cancer \\ & $\mathrm{PSA}=$ prostate-specific antigen \\ & $\mathrm{QOL}=$ quality of life \\ & $\mathrm{RP}=$ radical prostatectomy \\ & 3DMB $=$ three-dimensional pathologic mapping biopsy \end{aligned}

